\title{
Análise da espessura macular pela tomografia de coerência óptica no edema macular refratário de origem diabética após aplicação de triancinolona intravítrea
}

\author{
Macular thickness analysis by optic coherence tomography in refractory diabetic \\ macular edema treated with intravitreous triamcinolone
}

\author{
Alexandre Pinheiro ${ }^{1}$ \\ Pedro Durães Serracarbassa ${ }^{2}$ \\ Otacílio Oliveira Maia $\mathbf{J r}^{3}{ }^{3}$ \\ Walter Yukihiko Takahashi ${ }^{4}$
}

Trabalho realizado no Departamento de Retina e Vítreo do Serviço de Oftalmologia da Faculdade de Medicina da Universidade de São Paulo - USP - São Paulo (SP) Brasil.

${ }^{1}$ Aluno da Pós-Graduação (Doutorado) do Setor de Retina e Vítreo do Hospital das Clínicas da Faculdade de Medicina da Universidade de São Paulo - USP - São Paulo (SP) - Brasil.

2 Doutor em medicina, médico colaborador do Setor de Retina e Vítreo do Hospital das Clínicas da Faculdade de Medicina da USP - São Paulo (SP) - Brasil.

Aluno da Pós-Graduação (Doutorado) do Setor de Retina e Vítreo do Hospital das Clínicas da Faculdade de Medicina da USP - São Paulo (SP) - Brasil.

${ }^{4}$ Chefe do Setor de Retina e Vítreo, aluno da Pós-Graduação (Doutorado) do Setor de Retina e Vítreo do Hospital das Clínicas da Faculdade de Medicina da USP - São Paulo (SP) - Brasil.

Endereço para correspondência: Alexandre Pinheiro. Rua José Alves, 522 - Vitória (ES) CEP 29075-080

E-mail: Pinheiroalx@uol.com.br

Recebido para publicação em 17.10.2005

Última versão recebida em 18.06.2007

Aprovação em 22.06.2007

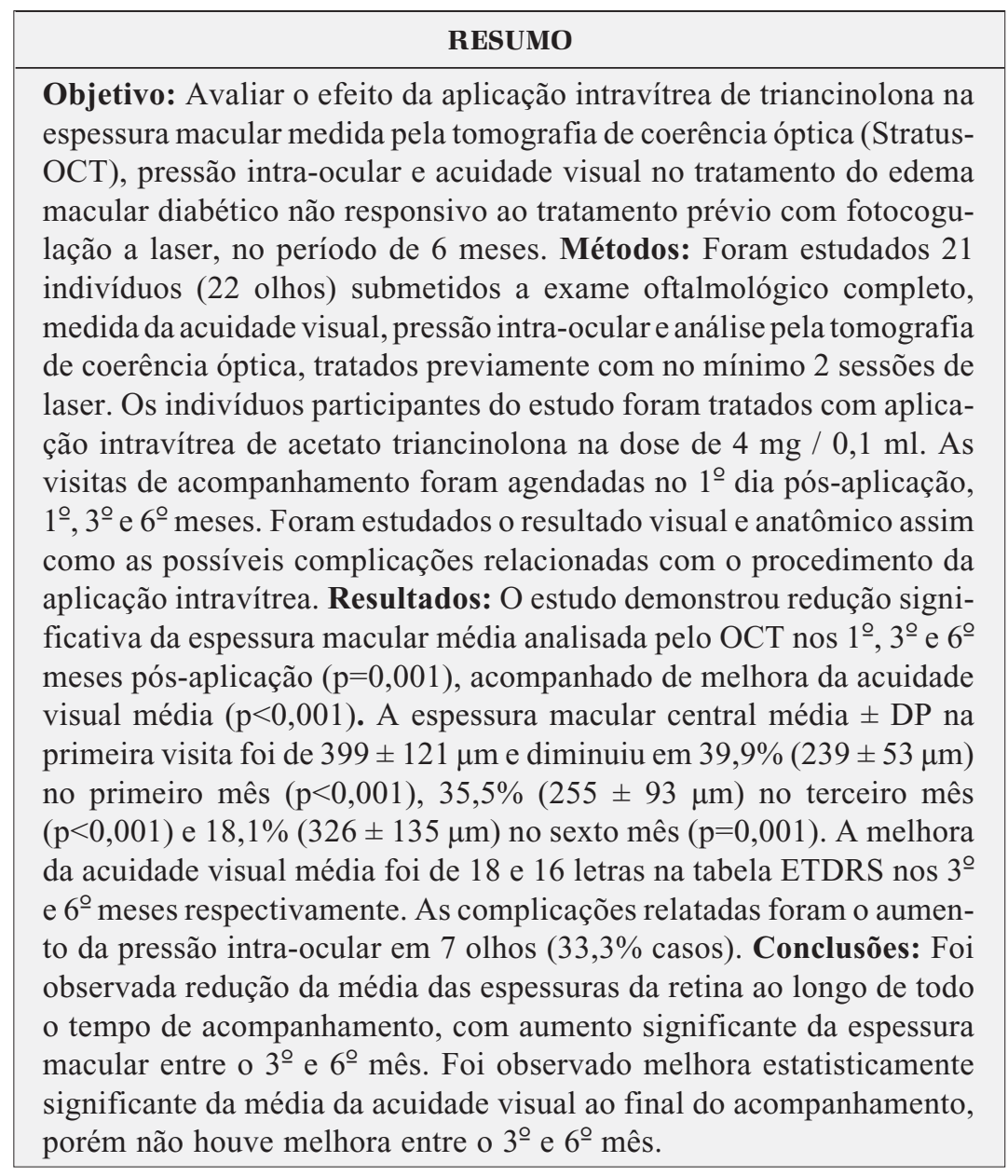

Descritores: Retinopatia diabética/complicações; Edema macular cistóide/etiologia; Triancinolona/administração \& dosagem; Injeções; Corpo vítreo; Tomografia de coerência óptica; Macula lútea; Acuidade visual

\section{INTRODUCÃO}

A retinopatia diabética é uma das complicações microvasculares mais comuns do diabetes. É a principal causa de cegueira irreversível em adultos 
com menos de 60 anos na população norte-americana ${ }^{(1)}$, apesar da terapia com controle glicêmico ou da fotocoagulação a laser da retina.

O edema macular é responsável por significativa perda de visão nos pacientes diabéticos. Os dados do "Wisconsin Epidemiologic Study of Diabetic Retinopathy" (WESDR) - com sua população formada por diabéticos tipo 1 usuários de insulina e diagnosticados com idade inferior a 30 anos indicaram uma incidência de edema macular entre $13,9 \%$ e $25,4 \%$ em $10 \operatorname{anos}^{(2)}$, e de $26 \%$ em $14 \operatorname{anos}^{(3)}$.

O "Early Treatment Diabetic Retinopathy Study" (ETDRS $)^{(4)}$ demonstrou o beneficio significante do tratamento com fotocoagulação a laser para o tratamento do edema macular clinicamente significativo. A fotocoagulação imediata reduziu a perda visual moderada em $50 \%$, porém $12 \%$ desses pacientes tratados perderam 15 ou mais letras na tabela ETDRS no seguimento de 3 anos e em menos de 3\% dos olhos houve melhora significante. Outro dado mostra que, $24 \%$ dos pacientes tratados tiveram aumento da espessura envolvendo o centro da mácula, nos 36 meses de seguimento. Isso sugere a existência de um subgrupo distinto de olhos com edema macular diabético resistente à terapia convencional e com pequeno ganho de visão após a terapia com laser ${ }^{(4)}$.

O acetato de triancinolona é uma suspensão de corticosteróide, não solúvel em água, que tem sido objeto de estudos recentes demonstrando efeito benéfico no tratamento de diversas doenças retinianas como: neovascularização de coróide $^{(5-6)}$, retinopatia diabética proliferativa ${ }^{(7)}$, telangiectasia justafoveal $^{(8)}$, oclusão de veia central da retina ${ }^{(9-10)}$, edema difuso refratário em retinopatia diabética ${ }^{(11)}$, uveites $^{(12-16)}$, edema cistóide do pseudofácico ${ }^{(17)}$ e visibilização intra-operatória da hialóide posterior ${ }^{(18)}$.

As hipóteses aventadas em relação ao seu mecanismo de ação incluem a ação antiinflamatória ${ }^{(19-20)}$, inibição do fator de crescimento vascular endotelial ${ }^{(21)}$, melhora da difusão ${ }^{(22)}$, e restabelecimento da barreira hemato-retiniana pela redução na permeabilidade ${ }^{(23-24)}$

O objetivo do presente estudo é avaliar o efeito da injeção intravítrea de triancinolona na espessura macular retiniana medida pela tomografia de coerência óptica (Stratus-OCT), pressão intra-ocular e acuidade visual no tratamento do edema macular diabético não responsivo ao tratamento prévio com fotocoagulação a laser, no período de 6 meses.

\section{MÉTODOS}

O estudo foi realizado no departamento de Retina da Clínica Oftalmológica da Universidade de São Paulo (USP). O estudo foi prospectivo, tipo série de casos com intervenção. Foram incluídos 21 indivíduos (22 olhos) que foram acompanhados por seis meses (julho de 2004 a janeiro de 2005). Dois sujeitos foram excluídos, sendo um no terceiro mês e outro no sexto mês, por não terem comparecido na data do acompanhamento determinado pelo estudo.
De acordo com o sexo, $8(36,4 \%)$ eram do sexo masculino e $14(63,6 \%)$ do feminino. Quatorze $(63,6 \%)$ eram brancos, 7 $(31,8 \%)$ negros e $1(4,5 \%)$ pardo. A idade variou entre 57 e 72 anos de idade, média \pm DP de $66,4( \pm 4,0)$.

Os critérios de inclusão foram: 1) indivíduos diabéticos com diagnóstico de edema macular difuso clinicamente significativo (EMCS) tratados previamente com pelo menos duas sessões de laser baseado nos critérios determinados pelo ETDRS $^{(3)}$, sem sucesso e com no mínimo três meses de intervalo, 2) ausência de glaucoma prévio e 3) meios oculares transparentes. Os critérios de exclusão foram: 1) indivíduos não tratados previamente com laser, 2) diagnóstico prévio de glaucoma, 3) catarata, 4) hemorragia vítrea e 5) retinopatia diabética proliferativa.

Os indivíduos foram submetidos a exame oftalmológico completo com medida da acuidade visual em logMAR, segundo a tabela do ETDRS $^{(3)}$, biomicroscopia estereoscópica e classificados de acordo com o critério determinado pelo $\operatorname{ETDRS}^{(3)}$, foi realizada medida da pressão intra-ocular (PIO) com tonômetro de aplanação e análise pela tomografia de coerência óptica (Stratus OCT-3, Carl Zeiss Meditec Inc. Dublin/CA, USA). A estratégia utilizada foi a análise macular utilizando-se o mapa da espessura macular (macular thickness map), sendo analisada a região central do mapa. Todos os indivíduos foram submetidos a três exames realizados pelo mesmo examinador. A melhor medida da espessura macular era obtida seguindo os seguintes critérios: 1) melhor nota dada pelo software para o scan obtido, 2) melhor conjunto de 6 scans com menor índice de erro na análise da espessura retiniana e 3) mapa melhor centrado na depressão foveal, caso presente.

Todos os exames pré-operatórios foram realizados no mesmo dia da aplicação intravítrea. A aplicação foi feita com base na diretriz publicada previamente ${ }^{(25)}$, com utilização de luvas estéreis, blefarostato, aplicação de iodo povidona, colírio anestésico tópico com agulha de insulina (30 gauge) no sítio temporal inferior sem realização de paracentese de câmara anterior. Foi utilizada a dose de $4 \mathrm{mg} / 0,1 \mathrm{ml}$ de acetato de triancinolona Kenalog ${ }^{\circledR} 40 \mathrm{mg} / \mathrm{ml}$ em todos sujeitos.

As visitas foram agendadas no primeiro dia pós-injeção, $1^{\circ}$, $3^{\circ}$ e $6^{\circ}$ meses. A cada visita era medida a PIO, acuidade visual ( $3^{\circ}$ e $6^{\circ}$ meses) e obtido o mapa macular (Stratus-OCT). O resultado visual e anatômico foi observado assim como as possíveis complicações relacionadas com o procedimento da aplicação intravítrea.

O estudo foi aprovado pelo Comitê de Ética da instituição e todos os indivíduos assinaram previamente o termo de consentimento livre esclarecido.

\section{RESULTADOS}

A acuidade visual média teve melhora de 18 e 16 letras na escala ETDRS (logMAR), no $3^{\circ}$ e $6^{\circ}$ meses do seguimento, respectivamente. $\mathrm{O} 3^{\circ}$ mês não apresentou diferença significativa em comparação com o $6^{\circ}$ mês $(p=0,351)$. 
Através da análise de variância com medidas repetidas observou-se que houve alteração significativa ao longo das avaliações ( $p=0,001)$. Ocorrendo melhora significativa entre a pré-aplicação e o $3^{\circ}$ mês $(\mathrm{p}<0,001)$, e a pré-aplicação e o $6^{\circ}$ mês $(\mathrm{p}<0,001)$ (Gráfico 1).

A espessura macular retiniana $(\mathrm{EM})$ média \pm DP prétratamento foi de $399( \pm 121 \mu \mathrm{m})$ para os 20 olhos analisados. No $1^{\circ}$ mês a melhora foi de $39,9 \%(239 \pm 53 \mu \mathrm{m})$; no $3^{\circ}$ mês foi de $35,9 \%(255 \pm 93 \mu \mathrm{m})$; no $6^{\circ}$ mês foi de $18,1 \%$ (326 $\pm 135 \mu \mathrm{m})$ (Gráfico 2).

No presente estudo foi observado que houve alteração significante das médias da EM ao longo das avaliações. Do prétratamento para o $1^{\circ}$ mês $(\mathrm{p}<0,001), 3^{\circ}$ mês $(\mathrm{p}<0,001)$ e para o $6^{\circ}$ mês $(p=0,001)$. A média do $1^{\circ}$ mês não difere significantemente do $3^{\circ}$ mês $(p=0,341)$ e difere do $6^{\circ}$ mês $(p=0,003)$. A média do $3^{\circ}$ mês apresenta diferença significante do $6^{\circ}$ mês $(\mathrm{p}=0,001)$. Isso demonstra piora significante na espessura entre o $3^{\circ}$ e $6^{\circ}$ mês, mas ainda assim, mantendo melhora significante entre o pré e o $6^{\circ}$ mês $(\mathrm{p}=0,001)$.

Com o objetivo de determinar se há correlação entre espessura macular pré-tratamento e evolução da acuidade visual e

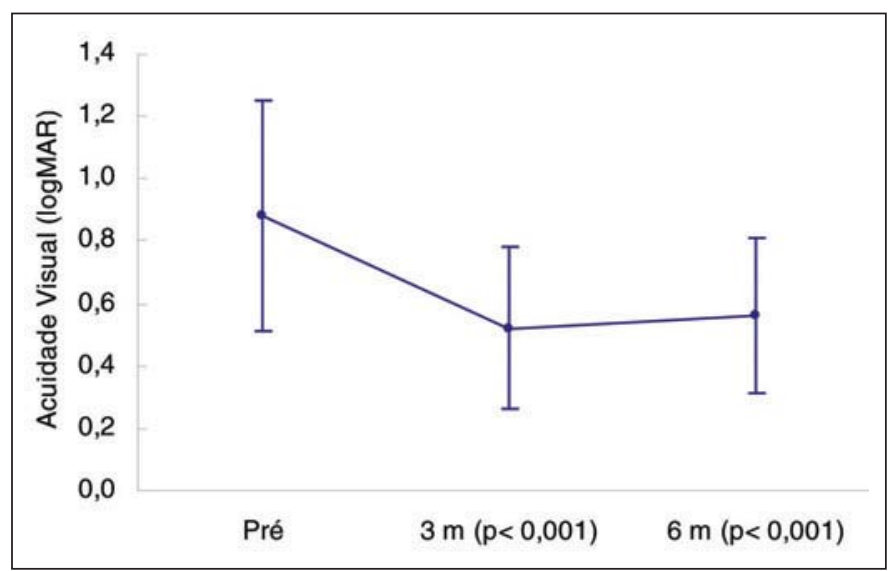

Gráfico 1 - Acuidade visual média (logMAR) durante o tempo de acompanhamento do estudo $(p=0,001)$

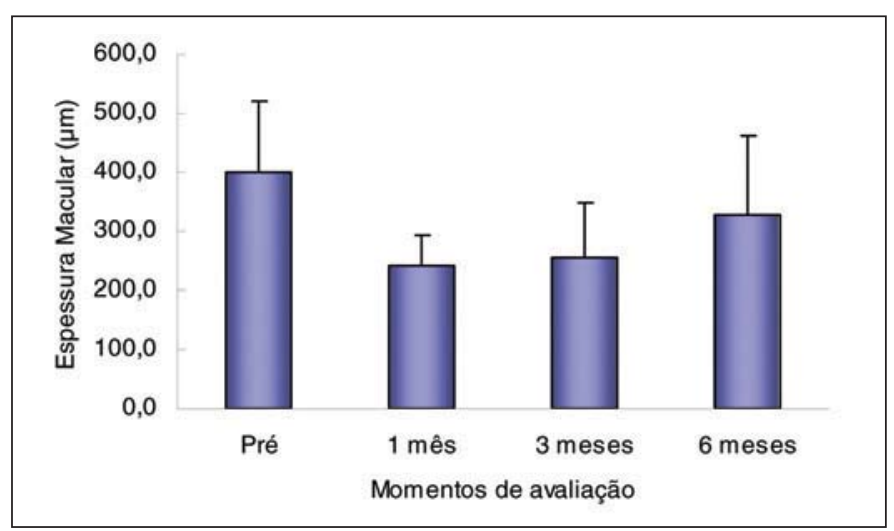

Gráfico 2 - Espessura macular média $(\mu \mathrm{m})$ dos pacientes durante o estudo $(p<0,001)$ espessura macular. Avaliamos separadamente dois grupos pela espessura macular central pré-tratamento: Grupo 1: EM $<400$ micra (n:12) e Grupo 2: EM $\geq 400$ micra (n:8). Os grupos não diferiram na acuidade visual média durante o acompanhamento do estudo $(p=0,691)$. No terceiro mês o grupo 1 obteve melhora na acuidade visual média de $46,9 \%$, e o grupo 2 $31,6 \%$, porém não houve diferença significante $(\mathrm{p}=0,417)$. No sexto mês a melhora foi de $38,2 \%$ no grupo 1 e de $31,6 \%$ no grupo $2(\mathrm{p}=0,908)$ (Tabela 1$)$.

O presente estudo observou que houve diferença significante entre os grupos em relação à espessura macular central média ao longo das avaliações $(\mathrm{p}<0,001)$. O grupo 1 apresentou alteração significante ao longo das avaliações $(p=0,002)$. A EM média pré-aplicação diferiu significativamente do $1^{\circ}$ mês $(p<0,001)$, do $3^{\circ}$ mês $(p=0,002)$ e do $6^{\circ}$ mês $(p=0,004)$, com decréscimo significante nestes períodos. Já o grupo 2 apresentou alteração significante somente durante as duas primeiras visitas $\left(1^{\circ}\right.$ mês e $3^{\circ}$ mês $)$. A EM média pré-aplicação diferiu significativamente do $1^{\circ}$ mês $(p<0,001)$ e do $3^{\circ}$ mês $(p=0,002)$, com decréscimo significante nestes períodos. Contudo, no $6^{\circ}$ mês não houve melhora significante $(p=0,076)$ (Tabela 2, Gráfico 3).

As complicações relatadas foram aumento da PIO em três olhos, acima de $21 \mathrm{mmHg}(22,23$ e $24 \mathrm{mmHg}-1$ dia, 1 mês, 3 mês) e quatro olhos tiveram aumento de $33 \%$ acima da PIO inicial durante o estudo, portanto, em 7 (33,3\%) olhos ocorreu acréscimo de $30 \%$ ou mais do valor da PIO pré-aplicação em algum momento das avaliações. Os três olhos que tiveram aumento acima de $21 \mathrm{mmHg}$ foram tratados com sucesso somente com tratamento clínico (colírio timolol 0,5\%) (Gráfico 4). Um indivíduo necessitou paracentese imediatamente após a injeção por ter tido aumento abrupto da PIO (50

\begin{tabular}{|c|c|c|c|}
\hline & Momentos & Média & DP \\
\hline $\begin{array}{l}\text { Grupo } 1(\mathrm{n}: 12) \\
<400 \mu \mathrm{m}\end{array}$ & $\begin{array}{c}\text { Pré-aplicação } \\
3 \text { meses } \\
6 \text { meses }\end{array}$ & $\begin{array}{l}0,81 \\
0,43 \\
0,50\end{array}$ & $\begin{array}{l} \pm 0,42 \\
\pm 0,19 \\
\pm 0,20\end{array}$ \\
\hline $\begin{array}{l}\text { Grupo } 2(\mathrm{n}: 8) \\
\geq 400 \mu \mathrm{m}\end{array}$ & $\begin{array}{c}\text { Pré-aplicação } \\
3 \text { meses } \\
6 \text { meses }\end{array}$ & $\begin{array}{l}0,98 \\
0,67 \\
0,67\end{array}$ & $\begin{array}{l} \pm 0,28 \\
\pm 0,28 \\
\pm 0,29\end{array}$ \\
\hline
\end{tabular}

\begin{tabular}{|lccc|}
\hline \multicolumn{4}{|c|}{$\begin{array}{c}\text { Tabela 2. Espessura macular média }(\boldsymbol{\mu m}) \\
\text { o seguimento do estudo } \\
(\mathbf{p}<0,001)\end{array}$} \\
& Momentos & Média & DP \\
Grupo 1 $(\mathrm{n}: 12)$ & Pré & 318,67 & $\pm 61,57$ \\
$<400 \mu \mathrm{m}$ & 1 mês & 225,67 & $\pm 58,21$ \\
& 3 meses & 222,25 & $\pm 57,98$ \\
& 6 meses & 256,08 & $\pm 84,72$ \\
Grupo 2 (n:8) & Pré & 520,25 & $\pm 77,98$ \\
$\geq 400 \mu \mathrm{m}$ & 1 mês & 261,25 & $\pm 39,64$ \\
& 3 meses & 305,63 & $\pm 115,87$ \\
& 6 meses & 433,25 & $\pm 129,23$
\end{tabular}




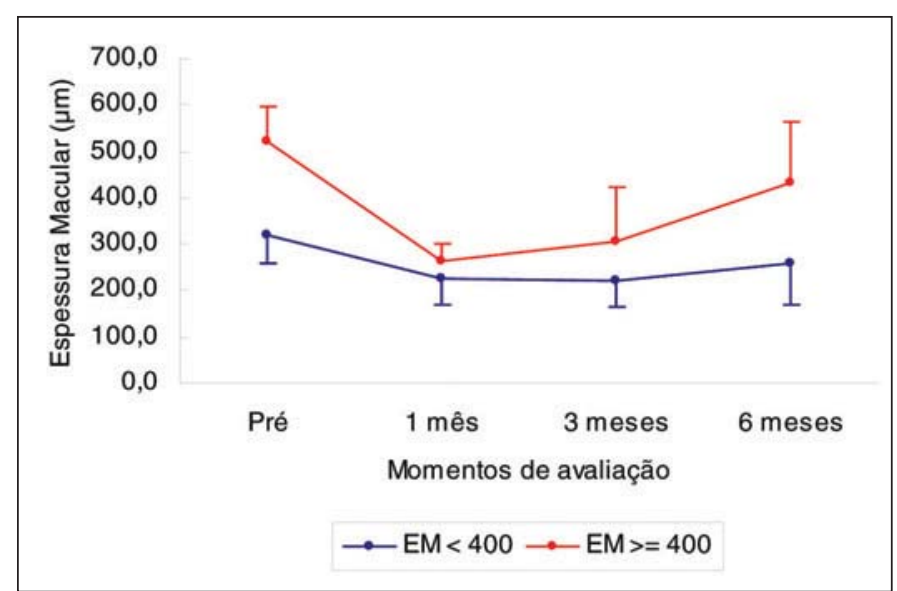

Gráfico 3 - Variação da espessura macular média $(\mu \mathrm{m})$ nos grupo 1 $(E M<400 \mu \mathrm{m})$ e grupo $2(E M \geq 400 \mu \mathrm{m})(p=0,691)$

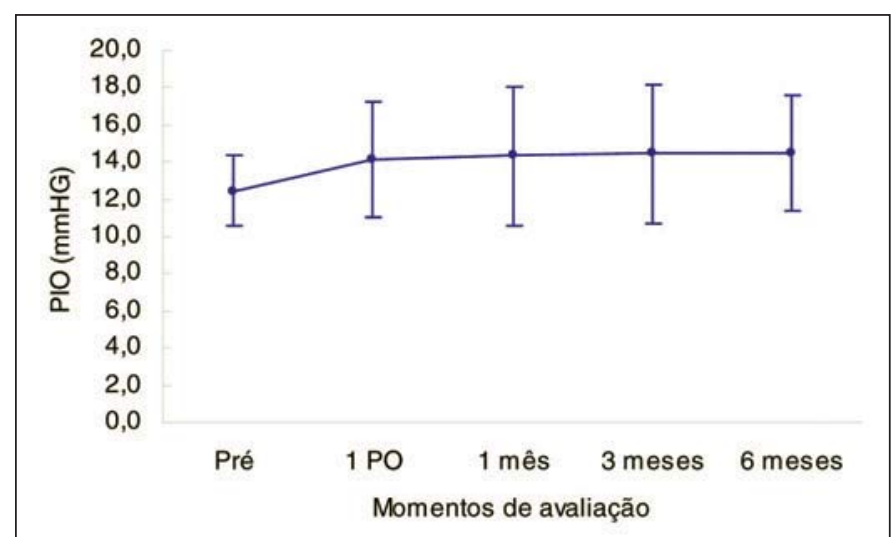

Gráfico 4 - Variação média da PIO durante o estudo

mmHg) e diminuição na perfusão do nervo óptico. Após a paracentese a PIO retornou ao normal e a perfusão do nervo óptico foi restabelecida. Nenhum caso evoluiu com catarata, hemorragia vítrea, endoftalmite ou pseudo-endoftalmite durante o tempo de acompanhamento.

\section{DISCUSSÃO}

Vários estudos relatam que o edema macular diabético tem melhora inicial significante, demonstrada pela tomografia de coerência óptica, após injeção intravítrea de triancinolona $^{(7,9,26-30)}$. A redução da espessura macular central e melhora da acuidade visual pode começar nas primeiras 48 horas $^{(29)}$. A questão atual mais importante relacionada à triancinolona intravítrea está na sua segurança e indicação precisa ${ }^{(25)}$.

A segurança da aplicação intravítrea da triancinolona foi investigada em modelos animais e alguns estudos demonstram uma baixa toxicidade com doses entre 1 e $8 \mathrm{mg}^{(31-33)}$. Altas doses de triancinolona ( 8 a $25 \mathrm{mg}$ ) parecem ser bem toleradas em humanos ${ }^{(34)}$. A farmacocinética da administração de triancinolona intravítrea depende da história de vi- trectomia prévia e da presença ou não do cristalino ${ }^{(26-27)}$. No modelo experimental em coelhos ${ }^{(26)}$, a sua meia-vida é mais curta em olhos com vitrectomia e lensectomia (6,5 dias), seguido por olhos somente vitrectomizados (18 dias), e por último nos olhos normais (41 dias). O primeiro estudo realizado em humanos utilizando uma dose de $4 \mathrm{mg}$ intravítrea demonstra que a meia-vida nos olhos não vitrectomizados foi de 18,6 dias e no olho vitrectomizado foi de 3,2 dias, o estudo estima que a triancinolona intravítrea pode estar presente no olho por até 3 meses (93 \pm 28 dias) após a aplicação ${ }^{(35)}$.

Alguns autores têm relatado uma necessidade de repetidas aplicações para o controle do edema macular diabético ${ }^{(25)}$, sendo o retratamento realizado entre três e seis meses. Estudos recentes demonstram que $90 \%$ dos olhos têm edema macular recorrente no $6^{\circ}$ mês de acompanhamento ${ }^{(28-30)}$. Os estudos de alguns autores sugerem que o efeito na acuidade visual parece ser mais duradouro, havendo a perda de uma linha de visão no $6^{\circ}$ mês de acompanhamento ${ }^{(29,36)}$. O presente estudo observou uma redução significante da espessura macular média no $1^{\circ}$ e $3^{\circ}$ meses pós-aplicação $(\mathrm{p}<0,001)$, o estudo atual também demonstrou que no $6^{\circ}$ mês ocorreu aumento significante da espessura retiniana média em relação ao $3^{\circ}$ mês $(p=0,001)$. A acuidade visual média obteve melhora significante no terceiro $(18$ letras ETDRS) $(p<0,001)$ e no sexto mês (16 letras ETDRS) $(\mathrm{p}<0,001)$.

Estudo realizado pelo grupo "Diabetic Retinopathy Clinical Research Network"(37) em pacientes com edema macular diabético sugere que há uma modesta correlação entre a espessura macular central medida pelo OCT e acuidade visual, havendo uma grande variação da acuidade visual para uma determinada faixa de espessura retiniana. Vários sujeitos com espessura macular aumentada obtiveram excelente acuidade visual e outros com a espessura macular normal eram portadores de baixa visão. Esses resultados sugerem que a análise da espessura macular retiniana pode não ser um bom substituto para a medida da acuidade visual.

O presente estudo analisou dois grupos separadamente pela espessura macular central pré-tratamento, Grupo 1: EM $<400$ micra (n:12) e Grupo 2: EM $\geq 400$ micra (n:8). A acuidade visual média não diferiu durante o acompanhamento do estudo comparando-se os dois grupos $(\mathrm{p}=0,691)$. O Grupo 2 com maior espessura macular obteve melhora significante da acuidade visual média no sexto mês $(\mathrm{p}=0,908)$, mesmo não havendo melhora significante da espessura macular média entre o terceiro $(305 \pm 115)$ e sexto mês $(433 \pm$ 129), $(\mathrm{p}=0,076)$. Os resultados do estudo atual são similares aos de Browning et al. (3), havendo, portanto uma discrepância entre a espessura retiniana e acuidade visual. É possível que a acuidade visual seja afetada por diversos fatores e variáveis, como a integridade do segmento externo dos fotorreceptores e a presença de isquemia foveolar, numa relação complexa e ainda não totalmente esclarecida.

Ainda devemos considerar que a análise da espessura macular pode ser subestimada, pois o programa de análise das imagens do Stratus-OCT tem algumas falhas notáveis. A 
maior delas é que o programa não analisa a verdadeira medida anatômica da espessura retiniana, devido a sua inabilidade para discriminar a junção do epitélio pigmentado com a camada de fotorreceptores ${ }^{(38)}$.

Recente estudo controlado e randomizado com dois anos de acompanhamento ${ }^{(39)}$ observou que o tratamento com a triancinolona intravítrea reduziu a espessura macular e melhorou a visão após tratamentos repetidos ao longo de todo o período de acompanhamento do estudo. Uma melhora $\geq 5$ letras foi observada em $56 \%$ dos indivíduos no grupo tratado, comparado a $26 \%$ no grupo placebo $(\mathrm{p}=0,006)$. A média de tratamentos foi de 2,6 no grupo tratado (n:34) e de 1,8 no grupo placebo (n:35). Outro estudo não controlado com dois anos de acompanhamento utilizando a dose de $4 \mathrm{mg}$ intravítrea $^{(40)}$, observou uma diferença significante da espessura macular central (OCT) entre o pré-tratamento e o $3^{\circ}$ dia, $1^{\circ}$, $3^{\circ}, 6^{\circ}, 9^{\circ}, 12^{\circ}, 18^{\circ}$ e $24^{\circ}$ meses pós-aplicação $(\mathrm{p}<0,001)$. Porém, a acuidade visual média (logMAR) obteve melhora significante somente no $1^{\circ}$ e $3^{\circ}$ meses de acompanhamento $(\mathrm{p}<0,05)$, as visitas entre o $6^{\circ}, 24^{\circ}$ meses não obtiveram melhora significante da acuidade visual média $(\mathrm{p}>0,05)$. Ocorreu a necessidade de reaplicação intravítrea em 29 $(38,7 \%)$ indivíduos do estudo.

O aumento da pressão intra-ocular parece não estar associado à dose da triancinolona intravítrea. Recente estudo utilizando doses entre 2 e $13 \mathrm{mg}$ não observou diferença estatisticamente significante entre os grupos estudados $(\mathrm{p}=0,77)^{(41)}$. Outros autores sugerem também que o aumento da pressão intra-ocular não ocorre nas aplicações repetidas subseqüentes caso ela não se eleve na primeira aplicação ${ }^{(7)}$. O presente estudo observou o aumento da PIO em 3 olhos, acima de $21 \mathrm{mmHg}$ e 4 olhos tiveram aumento de $33 \%$ acima da PIO inicial durante o estudo, portanto, em 7 (33,3\%) olhos ocorreu acréscimo de $30 \%$ ou mais do valor da PIO préaplicação durante as avaliações. Os três olhos que tiveram aumento acima de $21 \mathrm{mmHg}$ foram tratados com sucesso somente com tratamento clinico.

A efetividade da aplicação intravítrea de triancinolona foi comparada à aplicação subtenoniana em dois estudos pré$\operatorname{vios}^{(28,30)}$. Os estudos demonstram que a melhora na espessura macular e na acuidade visual são significativamente melhor no grupo da aplicação intravítrea e somente até o terceiro mês de acompanhamento. O estudo de Cardillo et al. também sugere que não há diferença significativa no aumento da PIO entre os grupos de aplicação intravítrea e de aplicação subtenoniana ${ }^{(30)}$.

\section{CONCLUSÃO}

Tendo em vista os resultados obtidos neste estudo, e levando-se em conta as limitações em relação à amostra avaliada, concluiu-se que a triancinolona intravítrea parece ser um tratamento promissor. E que o efeito de uma aplicação intravítrea na acuidade visual e espessura macular é limitado em curto prazo.
Os resultados de futuros estudos prospectivos em andamento nos ajudaram determinar a dosagem ideal e um esquema de reaplicações, ou ainda a possível utilização dos implantes de liberação lenta que estão em desenvolvimento.

\section{ABSTRACT}

Purpose: To assess the effect of intravitreal injections of triamcinolone on macular thickness (Stratus-OCT), intraocular pressure and visual acuity, in the treatment of diabetic macular edema non-responsive to previous laser photocoagulation, over a 6-month period. Methods: The study included 21 subjects (22 eyes) who underwent complete ophthalmologic examination, measurements of visual acuity and intraocular pressure, and OCT, previously treated with at least 2 laser applications. The study participants were treated with $4 \mathrm{mg} / 0.1 \mathrm{ml}$ intravitreal triamcinolone. Follow-up visits were scheduled for the $1^{\text {st }}$ day after treatment, and after 1,3 and 6 months. Visual and anatomical results, as well as possible complications associated with the intravitreal injection procedure were studied. Results: The study showed a significant reduction in mean macular thickness, assessed by OCT at 1,3 and 6 months post-treatment $(p=0.001)$, combined with an improvement of the mean visual acuity $(\mathrm{p}<0.001)$. Central macular thickness mean $\pm \mathrm{SD}$ value on the first visit was $399( \pm 121 \mu \mathrm{m})$; it was reduced by $39.9 \%(239 \pm$ $53 \mu \mathrm{m})$ at the first month $(\mathrm{p}<0.001), 35.5 \%(255 \pm 93 \mu \mathrm{m})$ at the third month $(\mathrm{p}<0.001)$, and $18.1 \%(326 \pm 135 \mu \mathrm{m})$ at the sixth month $(\mathrm{p}=0.001)$. The improvement in mean visual acuity was of 18 and 16 letters on the ETDRS table, on the $3^{\text {rd }}$ and $6^{\text {th }}$ month, respectively. Reported complications were an increase in intraocular pressure in seven eyes $(33.3 \%$ of the cases). Conclusions: A reduction in mean retinal thickness was observed throughout the entire follow-up period, with significant increase in macular thickness between the $3^{\text {rd }}$ and $6^{\text {th }}$ month. The mean visual acuity showed statistically significant improvement at the end of the follow-up period, but no change was noticed between the $3^{\text {rd }}$ and $6^{\text {th }}$ month.

Keywords: Diabetic retinopathy/complications; Macular edema, cystoid/etiology; Triamcinolone/administration \& dosage; Injections; Vitreous body; Tomography, optical coherence; Macula lutea; Visual acuity

\section{REFERÊNCIAS}

1. Kempen JH, O’Colmain BJ, Leske MC, Haffner SM, Klein R, Moss SE, Taylor HR, Hamman RF; Eye Diseases Prevalence Research Group.The prevalence of diabetic retinopathy among adults in the United States. Arch Ophthalmol. 2004;122(4):552-6.

2. Klein R, Klein BEK, Moss SE, Cruickshanks KJ. The Wisconsin epidemiologic study of diabetic retinopathy. XV. The long term incidence of macular edema. Ophthalmology. 1995;102(1):7-16.

3. Klein R, Klein BE, Moss SE, Cruickshanks KJ. The Wisconsin Epidemiologic Study of Diabetic Retinopathy: XVII. The 14-year incidence and progression of diabetic retinopathy and associated risk factors in type 1 diabetes. Ophthalmology. 1998;105(10):1801-15. Comment in: Ophthalmology. 1998;105(10):1799-800. 
4. Treatment techniques and clinical guidelines for photocoagulation of diabetic macular edema. Early Treatment Diabetic Retinopathy Study Report Number 2. Early Treatment Diabetic Retinopathy Study Research Group. Ophthalmology. 1987;94(7):761-74.

5. Danis RP, Ciulla TA, Pratt LM, Anliker W. Intravitreal triamcinolone acetonide in exudative age-related macular degeneration. Retina. 2000;20(3):244-50.

6. Gillies MC, Simpson JM, Luo W, Penfold P, Hunyour AB, Chua W, et al. A randomized clinical trial of a single dose of intravitreal triamcinolone acetonide for neovascular age-related macular degeneration: one-year results. Arch Ophthalmol. 2003;121(5):667-73. Comment in: Arch Ophthalmol. 2004;122 (10:1571-2; author reply 1572

7. Jonas JB, Hayler JK, Sofker A, Panda-Jonas S. Intravitreal injection of crystalline cortisone as adjunctive treatment of proliferative diabetic retinopathy. Am J Ophthalmol. 2001;131(4):468-71.

8. Alldredge CD, Garretson BR. Intravitreal triamcinolone for the treatment of idiopathic juxtafoveal telangiectasis. Retina. 2003;23(1):113-6.

9. Greenberg PB, Martidis A, Rogers AH, Duker JS, Reichel E. Intravitreal triamcinolone acetonide for macular o edema due to central retinal vein occlusion. Br J Ophthalmol. 2002;86(2):247-8.

10. Ip MS, Kumar KS. Intravitreous triamcinolone acetonide as treatment for macular edema from central retinal vein occlusion. Arch Ophthalmol. 2002; 120(9):1217-9.

11. Martidis A, Duker JS, Greenberg PB, Rogers AH, Puliafito CA, Reichel E, et al. Intravitreal triamcinolone for refractory diabetic macular edema. Ophthalmology. 2002;109(5):920-7.

12. Andrade RE, Muccioli C, Farah ME. Injeção intra-vítrea de acetato de triancinolona no tratamento da síndrome de Vogt-Koyanagi-Harada. Arq Bras Oftalmol. 2004;67(3):401-6.

13. Godoy G, Finamor LP, Andrade R, Muccioli C. Triancinolona intra-vítrea em vasculite retiniana: relato de caso. Arq. Bras. Oftalmol. 2004;67(6):953-6.

14. Finamor LP, Dimantas MA, Campos VE, Prata Junior JÁ, Muccioli C. Efeitos da injeção subtenoniana posterior de corticóide em pacientes com uveíte. Arq Bras Oftalmol. 2003;66(3):289-91.

15. Antcliff RJ, Spalton DJ, Stanford MR, Graham EM, Ffytche TJ, Marshall J. Intravitreal triamcinolone for uveitis cystoid macular edema: An optical coherence tomography study. Ophthalmology. 2001;108(4):765-72.

16. Martidis A, Duker JS, Puliafito CA. Intravitreal triamcinolone for refractory cystoid macular edema secondary to birdshot retinochoroidopathy. Arch Ophthalmol. 2001;119(9):1380-3.

17. Conway MD, Canakis C, Livir-Rallatos C, Peyman GA. Intravitreal triamcinolone acetonide for refractory chronic pseudophakic cystoid macular edema. J Cataract Refract Surg. 2003;29(1):27-33

18. Peyman GA, Cheema R, Conway MD, Fang T. Triamcinolone acetonide as an aid to visualization of the vitreous and the posterior hyaloid during pars plana vitrectomy. Retina. 2000;20(5):554-5.

19. Floman N, Zor U. Mechanism of steroid action in ocular inflammation: Inhibition of prostaglandin production. Invest Ophthalmol Vis Sci. 1977;16(1):69-73.

20. Ishibashi T, Miki K, Sorgente N, Patterson R, Ryan SJ. Effects of intravitreal administration of steroids on experimental subretinal neovascularization in the subhuman primate. Arch Ophthalmol. 1985;103(5):708-11.

21. Bandi N, Kompella UB. Budesonide reduces vascular endothelial growth factor secretion and expression in airway (Calu-1) and alveolar (A549) epithelial cells. Eur J Pharmacol. 2001;425(2):109-16.

22. Sze PY, Iqbal Z. Glucocorticoid action on depolarization-dependent calcium influx in brain synaptosomes. Neuroendocrinology. 1994;59(5):457-65.

23. Wilson CA, Berkowitz BA, Sato Y, Ando N, Handa JT, de Juan E Jr. Treatment with intravitreal steroid reduces blood-retinal barrier breakdown due to retinal photocoagulation. Arch Ophthalmol. 1992;110(8):1155-9.

24. Penfold PL, Wen L, Madigan MC, King NJ, Provis JM. Modulation of per- meability and adhesion molecule expression by human choroidal endothelial cells. Invest Ophthalmol Vis Sci. 2002;43(9):3125-30.

25. Aiello LP, Brucker AJ, Chang S, Cunningham ET Jr, D‘Amico DJ, Flynn HW Jr, et al. Evolving guidelines for intravitreous injections. Retina. 2004;24(5 Suppl):S3-19.

26. Schindler RH, Chandler D, Thresher R, Machemer R. The clearance of intravitreal triamcinolone acetonide. Am J Ophthalmol. 1982;93(4):415-7.

27. Scholes GN, O'Brien WJ, Abrams GW, Kubicek MF. Clearance of triamcinolone from vitreous. Arch Ophthalmol. 1985;103(10):1567-9.

28. Bonini-Filho MA, Jorge R, Barbosa JC, Calucci D, Cardillo JA, Costa RA Intravitreal injection versus sub-Tenon's infusion of triamcinolone acetonide for refractory diabetic macular edema: a randomized clinical trial. Invest Ophthalmol Vis Sci. 2005;46(10):3845-9.

29. Patelli F, Fasolino G, Radice P, Russo S, Zumbo G, Di Tizio FM, et al. Time course of changes in retinal thickness and visual acuity after intravitreal triamcinolone acetonide for diffuse diabetic macular edema with and without previous macular laser treatment. Retina, 2005;25(7):840-5

30. Cardillo JA, Melo LA Jr, Costa RA, Skaf M, Belfort R Jr, Souza-Filho AA, et al. Comparison of intravitreal versus posterior sub-Tenon's capsule injection of triamcinolone acetonide for diffuse diabetic macular edema. Ophthalmology, 2005;112(9):1557-63

31. McCuen BW $2^{\text {nd }}$, Bessler M, Tano Y, Chandler D, Machemer R. The lack of toxicity of intravitreally administered triamcinolone acetonide. Am J Ophthalmol. 1981;91(6):785-8

32. Chandler DB, Rozakis G, de Juan E Jr, Machemer R. The effect of triamcinolone acetonide on a refined experimental model of proliferative vitreoretinopathy. Am J Ophthalmol. 1985;99(6):686-90.

33. Hida T, Chandler D, Arena JE, Machemer R. Experimental and clinical observations of the intraocular toxicity of commercial corticosteroid preparations. Am J Ophthalmol. 1986;101(2):190-5.

34. Ciulla TA, Criswell MH, Danis RP, Hill TE. Intravitreal triamcinolone acetonide inhibits choroidal neovascularization in a laser-treated rat model. Arch Ophthalmol. 2001;119(3):399-404.

35. Beer PM, Bakri SJ, Singh RJ, Liu W, Peters GB $3^{\text {rd }}$, Miller M. Intraocular concentration and pharmacokinetics of triamcinolone acetonide after a single intravitreal injection. Ophthalmology. 2003;110(4):681-6.

36. Larsson J, Zhu M, Sutter F, Gillies MC. Relation between reduction of foveal thickness and visual acuity in diabetic macular edema treated with intravitreal triamcinolone. Am J Ophthalmol. 2005;139(5):802-6.

37. Diabetic Retinopathy Clinical Research Network, Browning DJ, Glassman AR, Aiello LP, Beck RW, Brown DM, Fong DS, Bressler NM, Danis RP, Kinyoun JL, Nguyen QD, Bhavsar AR, Gottlieb J, Pieramici DJ, Rauser ME, Apte RS, Lim JI, Miskala PH. Relationship between optical coherence tomography-measured central retinal thickness and visual acuity in diabetic macular edema. Ophthalmology. 2007;114(3):525-36

38. Costa RA, Calucci D, Skaf M, Cardillo JA, Castro JC, Melo LA Jr, et al. Optical coherence tomography 3: Automatic delineation of the outer neural retinal boundary and its influence on retinal thickness measurements. Invest Ophthalmol Vis Sci. 2004;45(7):2399-406.

39. Gillies MC, Sutter FK, Simpson JM, Larsson J, Ali H, Zhu M. Intravitreal triamcinolone for refractory diabetic macular edema: two-year results of a double-masked, placebo-controlled, randomized clinical trial. Ophthalmology, 2006;113(9):1533-8

40. Batioglu F, Ozmert E, Parmak N, Celik S. Two-year results of intravitrea triamcinolone acetonide injection for the treatment of diabetic macular edema. Int Ophthalmol. 2007.[epub ahead of print]

41. Spandau UH, Derse M, Schimitz-Valckenberg P, Papoulis C, Jonas JB. Dosage dependency of intravitreal triamcinolone acetonide as treatment for diabetic macular oedema. Br J Ophthalmol. 2005;89(8):999-1003. 